\title{
Surface inspection via projection interferometry
}

\author{
Roger W. Wygant, Silverio P. Almeida, and Oliverio D. D. Soares
}

\begin{abstract}
Projection fringe interferometry provides a useful technique for nondestructive surface analysis. Two beam interferometric fringes were projected onto a block of aluminum whose surface had various types of grooves cut into it. The fringes were digitized and analyzed via an automatic fringe tracking algorithm. Threedimensional topographical maps of the surface's microstructure are presented together with a statistical analysis of surface parameters including average roughness, height distributions, and the autocorrelation function.
\end{abstract}

\section{Introduction}

The projection interferometer (PI) offers several advantages over the reflection type. Interference fringes are produced by intersecting two expanded collimated laser beams onto a test surface, rather than reflecting a plane wave from a test surface for subsequent interference with a plane wave reference. The projection fringe method makes it ideally suited to the study of optically rough and low reflectivity surfaces. In addition, the lateral and vertical resolutions of the instrument are variable over 6 orders of magnitude from subwavelength to the limits imposed by large aperture beam expanding optics. The interferograms characteristic of the PI typically consist of straight lines deviated by surface roughness. This type of pattern is more readily analyzed than a pattern containing closed loop fringes and does not present ambiguities as to the sign of the height deviations. The first PI arrangements proposed ${ }^{1-3}$ were shown to be equivalent to holographic techniques. ${ }^{4}$ The interference of two obliquely intersecting beams has been treated in explicit detail. 5 The theory, operational characteristics, and sources of error in the use of the projection interferometer are also noted in the literature. ${ }^{6-11}$

\section{Theory of Interferograms}

Two expanded collimated laser beams are allowed to intersect thereby resulting in a family of constructive and destructive interference planes. The constructed planes will be parallel to the bisector of the angle

Oliverio Soares is with University of Porto, Physics Department, 4000 Porto, Portugal; the other authors are with Virginia Polytechnic Institute \& State University, Physics Department, Blacksburg, Virginia 24061.

Received 17 March 1988.

0003-6935/88/224626-05\$02.00/0.

(c) 1988 Optical Society of America. between the two incident beams and perpendicular to the plane containing these beams. If we assume the two intersecting beams are uniform, equal in amplitude, frequency, and polarizations, and perfect plane wavefronts, the intensity of their intersecting volume will have the form

$$
I(\mathbf{x})=c \epsilon|E|^{2}\left[1+\cos \left[\left(\mathbf{k}_{1}-\mathbf{k}_{2}\right) \cdot \mathbf{x}\right]\right],
$$

where $c$ is the speed of light, $\epsilon$ is the permittivity of the propagation medium, $E$ is the electric field amplitude, and $\mathbf{k}_{i}$ are the wave vectors of the two beams. If the angle between the vectors $\mathbf{k}_{i}$ is $2 \phi$, and the wavelength of the radiation is $\lambda$, the spatial period of the interference fringe planes will be

$$
d=\lambda / 2 \sin \phi .
$$

When a test surface is placed in the volume of intersection, with its normal at an angle $\beta$ to the planes of interference, the spatial period of the fringes on the surface is

$$
\begin{aligned}
d_{\beta} & =d / \cos \beta \\
& =\lambda / 2 \sin \phi \cos \beta .
\end{aligned}
$$

If the surface is viewed at an angle $\gamma$ from the surface normal, the observed spatial period will be

$$
\begin{aligned}
d_{\gamma} & =d_{\beta} \cos \gamma \\
& =\lambda \cos \gamma / 2 \sin \phi \cos \beta .
\end{aligned}
$$

The angles $\beta$ and $\gamma$ are illustrated in Fig. 1. If there exists a height deviation $\Delta z\left(x_{1}, y_{1} ; x_{2}, y_{2}\right)$ between two points, the observed change in the fringe period between those points will be

$$
\delta_{\gamma}=-\Delta z\left(x_{1}, y_{1} ; x_{2}, y_{2}\right) \sin (\beta-\gamma) / \cos \beta
$$

This compares to the observed fringe spatial period $d_{\gamma}$ as

$$
\delta_{\gamma} / d_{\gamma}=-2 \Delta z \sin \phi \sin (\beta-\gamma) / \lambda \cos \gamma .
$$

Inverting this to obtain $\Delta z$ yields 


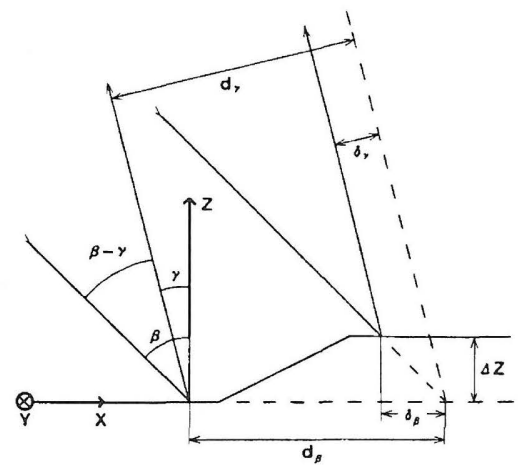

Fig. 1. Geometrical relationships between experimental parameters.

$$
\Delta z=-\lambda\left(\delta_{\gamma} / d_{,}\right) \cos \gamma / 2 \sin \phi \sin (\beta-\gamma) .
$$

Note that the surface should not be observed at an angle $\gamma=\beta$.

The minimum obtainable fringe spacing, from $\mathrm{Eq}$. (2), is $\lambda / 2$. However, to produce a meaningful interferogram, neither beam can cast a shadow on the test surface. Thus the minimum fringe spacing on a surface is dependent on the maximum slopes present on the surface. Due to the limitations of imaging optics, the minimum resolvable lateral distance for a given depth of focus $h$ is ${ }^{6}$

$$
d_{\min }=2 h_{1} \lambda /(8 h-\lambda) .
$$

If, on the right-hand side of Eq. (7), $\lambda$ is assumed to be known accurately, the angles are measured geometrically and $\delta_{\gamma}$ and $d_{\gamma}$ are measured experimentally in terms of digital pixels, the absolute error in $\Delta z$ will be given by

$$
\begin{aligned}
\epsilon(\Delta z) / \Delta z= & \epsilon\left(\delta_{,}\right) / \delta_{i}+\epsilon\left(d_{,}\right) / d, \\
& +\epsilon(\delta)|\cos (\beta-\gamma)-\tan \gamma| \\
& +\epsilon(\phi) \cos \phi \\
& +\epsilon(\phi) \cos (\beta-\gamma),
\end{aligned}
$$

where $\epsilon(x)$ represents the absolute error in the variable $x$.

\section{Analysis of the Interferograms}

Four basic steps are involved in the analysis of the projection interferograms: (1) noise reduction of the image; (2) construction of a phase map for the interferogram; (3) computation of the surface contours using the spatial deviations of phases in the interferogram from a computer-generated reference phase map representing an ideal flat surface; (4) statistical analysis and characterization of the surface structure.

There are three main sources of noise in the digitized interferograms. The highest frequency noise, with a delta function autocorrelation, arises from the digitization electronics. Speckle noise is present in all coherent imaging systems arising from random interference of the coherent radiation. The third type of noise is variations in the bias intensity and amplitude of the interference sinusoid due to local variations in the surface normal and reflectivity. Including terms for each type of noise, the digitized image can be described by the intensity surface:

$$
I(x, y)=S(x, y)\left\{A\left(x, y^{\prime}\right)+B\left(x, y^{\prime}\right) \cos \left[\Phi\left(x, y^{\prime}\right)\right]\right\}+E\left(x, y^{\prime}\right) .
$$

In this expression, $S(x, y)$ describes the speckle noise, $E(x, y)$ describes the electronic noise, and $A(x, y)$ and $B(x, y)$ arise from local changes in surface properties. The multiplicative affects of variations in surface properties on the bias intensity and amplitude of the interference sinusoid are displayed separately as $A(x, y)$ and $B(x, y)$ to facilitate the description of the affects of subsequent image processing operations on these quantities. The phase $\Phi(x, y)$ of the interference in the cosine term is the useful information in the image.

The electronic noise $E(x, y)$ is an additive zero mean noise unrelated to the interference pattern and could be properly eliminated by averaging several digitizations of the same image. However, it is just as easily dealt with in the same process as that used for suppressing the speckle noise $S(x, y)$. The analytical form of coherent speckle noise in an imaging system is that of a multiplicative noise with an inverse exponential autocorrelation function. The 1/e distance in the autocorrelation function characterizes the speckle size, which is determined by the structure of the test surface and the optical imaging system. ${ }^{12}$ In general, the frequency of the interference pattern must be lower than that of the speckle to obtain a usable interferogram. With this condition, both high frequency noises can be subdued through the convolution of the image with a smoothing function $W(x, y)$. This operation is represented as follows:

$$
\begin{aligned}
I^{\prime}(x, y)= & \sum_{\xi=x-\Delta x}^{x+\Delta x} \sum_{\eta=\gamma-\Delta y}^{y+\Delta y} W\left(\xi-x, \eta-y^{\prime}\right) I(\xi, \eta) \\
= & \sum_{\xi} \sum_{\eta} W\left(\xi-x, \eta-y^{\prime}\right)(S(\xi, \eta) \cdot\{A(\xi, \eta) \\
& +B(\xi, \eta) \cos [\Phi(\xi, \eta)]\}+E(\xi, \eta)) \\
\approx & \left\{A^{\prime}\left(x, y^{\prime}\right)+B^{\prime}\left(x, y^{\prime}\right) \cos \left[\Phi\left(x, y^{\prime}\right)\right]\right\} \\
& \cdot \sum_{\xi} \sum_{\eta} W\left(\xi-x, \eta-y^{\prime}\right) S(\xi, \eta) \\
& +\sum_{\xi} \sum_{\eta} W\left(-x,-y^{\prime}\right) E(\xi, \eta) \\
\propto & A^{\prime}(x, y)+B^{\prime}\left(x, y^{\prime}\right) \cos \left[\Phi\left(x, y^{\prime}\right)\right] .
\end{aligned}
$$

In this approximation, $W(x, y)$ is assumed to be a smooth function defined in the domain $x, \epsilon[-\Delta x, \Delta x], y$ $\epsilon[-\Delta y, \Delta y]$. As $E(x, y)$ is a high frequency zero mean function, its convolution with $W(x, y)$ should yield zero. The interference terms are removed from the summation under the assumptions that $\Delta x$ and $\Delta y$ are much smaller than the period of the cosine and that $A(x, y)$ and $B(x, y)$ are approximately constant throughout the averaging region. Note that the functions $A^{\prime}(x, y)$ and $B^{\prime}(x, y)$ will be high frequency filtered versions of $A(x, y)$ and $B(x, y)$, implying that high frequency effects of surface variations will be masked and 


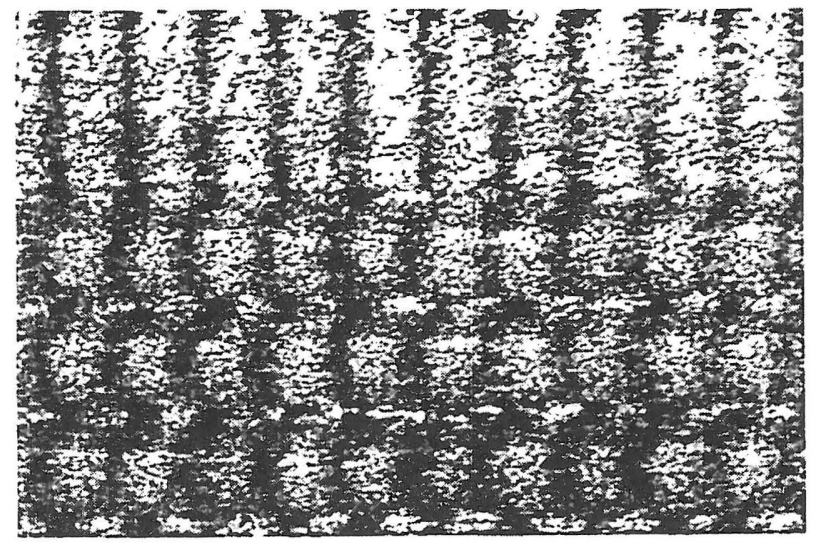

Fig. 2. Interference fringes projected onto an aluminum block with machined grooves.

the amplitude of the interference sinusoid will be decreased. The summation over $S(x, y)$ can then be taken to be constant as $S(x, y)$ is a high frequency function which has a uniform statistical description throughout the image, determined by the surface and observation optics. This yields the function $I^{\prime}(x, y)$, a biased sinusoid with varying amplitude and bias. To reduce the effect of bias variations, this image is then averaged within a region described by

$$
\left.(x, y) \epsilon|R| x=\delta_{A}, y_{\epsilon}[-p / 2, p / 2]\right\} \text {. }
$$

In this, $\delta_{x}$ is the Kronecker delta, $p$ is the period of the sinusoid, and it is assumed that the predominant direction of the fringes is parallel to the $x$ axis. The averaged intensity can then be represented by

$$
\begin{aligned}
I^{\prime \prime}(x, y) & =\sum_{(\xi, \eta) \cdot l^{\prime}}\left\{A^{\prime}(\xi, \eta)+B^{\prime}(\xi, \eta) \cos [\Phi(\xi, \eta)]\right\} \\
& \approx A^{\prime}\left(x, y^{\prime}\right) .
\end{aligned}
$$

This is true assuming $B^{\prime}(x, y)$ varies slowly, so that the sum over one period of the cosine term yields zero. This intensity is then subtracted from $I^{\prime}(x, y)$ to yield an unbiased sinusoid of varying amplitude. These amplitude variations can be easily accounted for in the phase calculations. Of course, these operations are based on rather broad assumptions as to the frequencies of different components of the digitized interferograms, but they generally result in vast improvements to the SNR of the interferograms.

After noise reduction, the phase of interference may be mapped. The initial step in this is to define the positions of the fringe extrema in the interferograms. This is done using a fringe tracking algorithm, which tabulates statistics characterizing the pattern as it operates. These statistics are used to define a neighborhood about a fringe line in which to search for a continuation of that fringe line. A quadratic fit is then performed within this neighborhood to define the next extreme point continuing the fringe. From the characteristics of the extrema pattern, a phase map representing an idealized flat surface can be generated by computer. The experimentally obtained phases are found by defining a local sinusoid between the extrema and using the intensity and slope at each pixel to define the phase at that pixel from the local sinusoid. To reduce the effects of noise, the phase along each column in the image is found traversing the column in each direction while requiring a monotonic change in each direction. The results from these two traversals are then averaged. At each pixel, the spatial deviation of that pixel's phase from the idealized pattern is calculated to yield $\delta$, of Eqs. (6) and (7). Equation (7) is then invoked to define the height variation at that pixel from the idealized flat surface.

To statistically characterize the surface structure the rms and average roughnesses of the surface, the skew and kurtosis of the height distribution, and the autocorrelation function of the surface are used. For an $N \times N$ pixel representation of the surface, the rms roughness $R_{k x}$ (which is equal to the standard deviation $\sigma$ of the height distribution) is given by

$$
R_{\mathrm{u}}=\left(N_{N^{2}}\left\{\sum_{i, j=1}^{N}[z(i, j)-\bar{z}]^{2}\right\}\right)^{1 / 2},
$$

where $z(i, j)$ is the height at the pixel $(i, j)$, and $\bar{z}$ is the average height of the surface.

The average roughness $R$ is given by

$$
R_{n}=\left[\sum_{i, j=1}|z(i, j)-\bar{z}|\right] \mid N^{2} .
$$

The skew of the height distribution, which is the third moment of the distribution, measuring its symmetry, is given by

$$
\text { skew }=\left(\left\{\sum_{i, l=1}^{N}[z(i, j)-\bar{z}]^{3}\right\} / N^{\prime}\right) / \sigma^{3} .
$$

The kurtosis, which is the fourth moment, measures the heaviness of the tails of the distribution. A Gaussian curve has a kurtosis of exactly 3.0. The formula for the kurtosis is

$$
\text { kurtosis }=\left(\left\{\sum_{i, j=1}^{N}[z(i, j)-\bar{z}]^{4}\right\} / N^{2}\right) / \sigma^{4} .
$$

The autocorrelation function measures the correlation in height of pixels at given lateral displacements from each other. It has the range $[-1,1]$, with the property that the point $(0,0)$ always has the value 1 . For $n$ samples, the autocorrelation function has the form

$$
C(x, y)=\frac{1}{n} \sum_{k=1}^{n}\left\{\left[z\left(i_{k}, j_{k}\right)-\bar{z}\right]\left[z\left(i_{k}+x, j_{k}+y\right)-\bar{z}\right] / \sigma^{2}\right\} .
$$

This set of surface characterizing statistics represents the most thorough description obtainable. ${ }^{9}$

\section{Results and Discussion}

The results presented here are from a grooved aluminum block milled with a slitting saw in the VPI\&SU Physics Department Machine Shop. A photograph of the surface with interference fringes projected onto it 


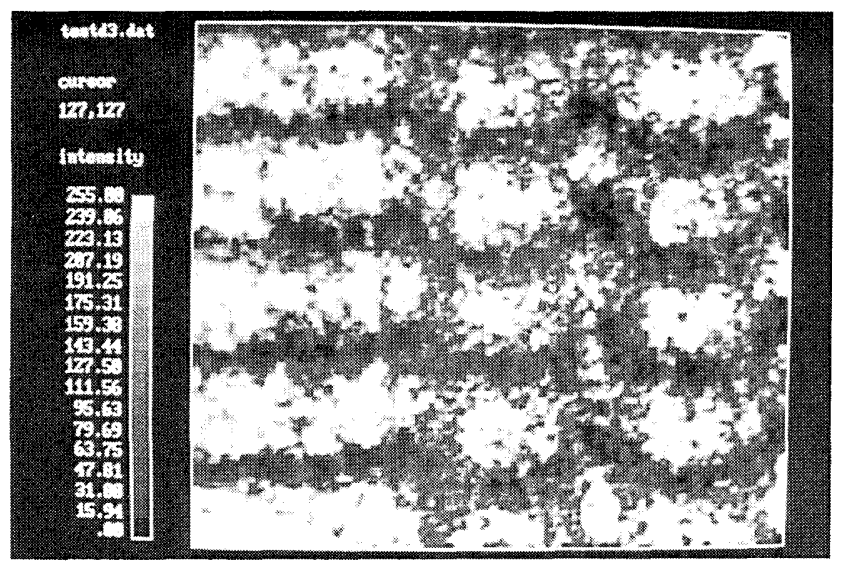

Fig. 3. Digital image obtained from the photograph of Fig. 2. Image is magnified and rotated $90^{\circ}$ counterclockwise relative to that of Fig. 2.

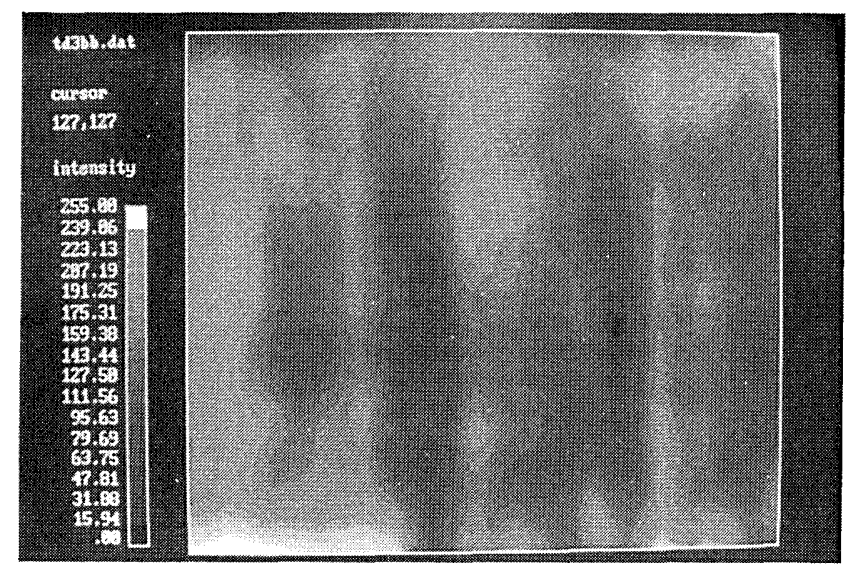

Fig. 4. Bias intensity of the interference obtained from image of Fig. 3.

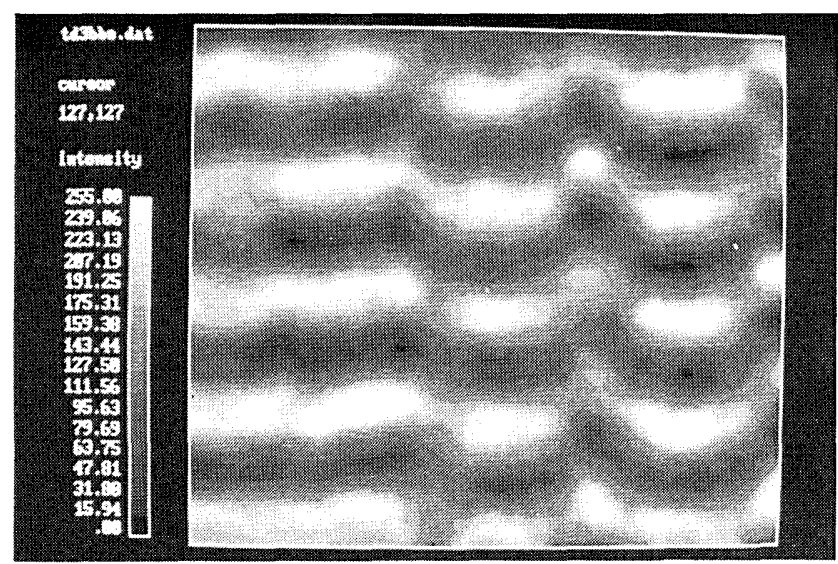

Fig. 5. Interferogram of Fig. 2 after noise reduction and bias intensity flattening.

is shown in Fig. 2. The spatial period of the fringes on the surface was $143.7 \mu \mathrm{m}$. Figure 3 shows an image digitized from the photograph in Fig. 2. The bias intensity of the interference, obtained by averaging the image of Fig. 3 with an operator whose length equaled the fringe period, is shown in Fig. 4. The interferogram of Fig. 5 is the result of noise suppression and bias

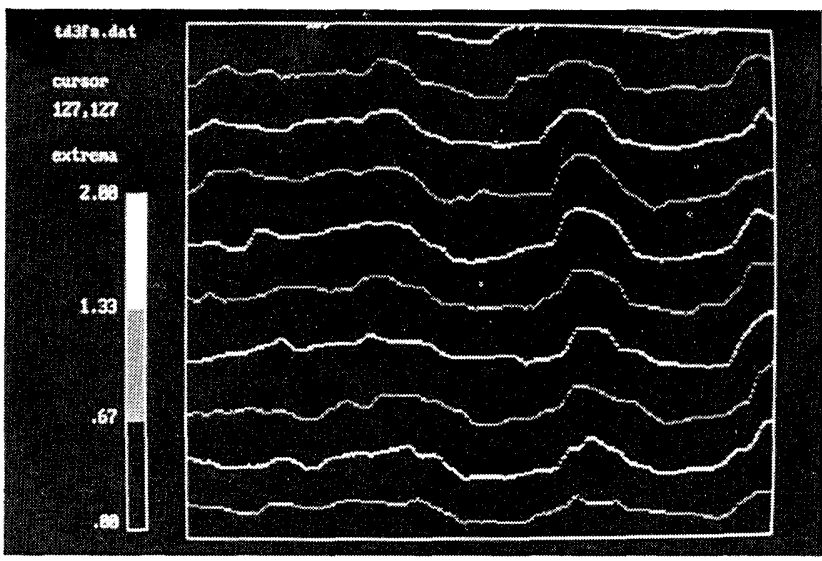

Fig. 6. Symbolic fringe extrema pattern derived from image of Fig. 5.

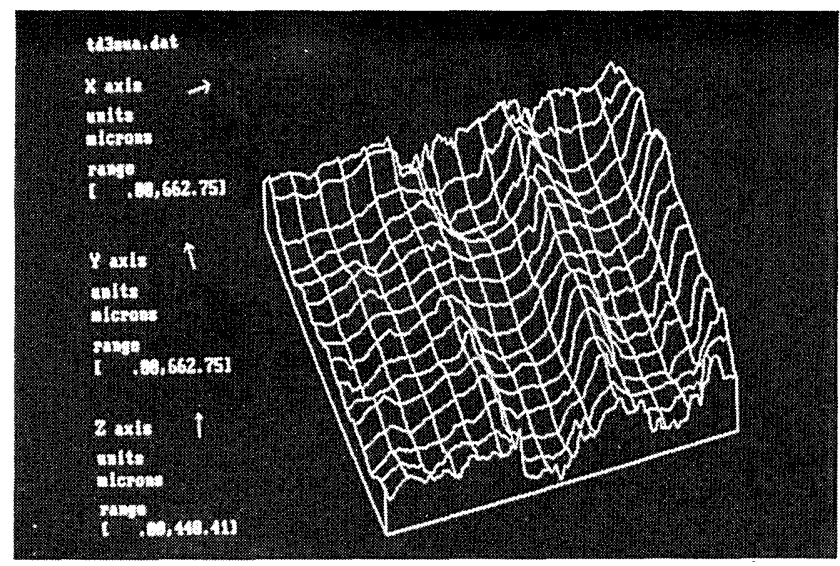

Fig. 7. Surface calculated from images of Figs. 5 and 6.

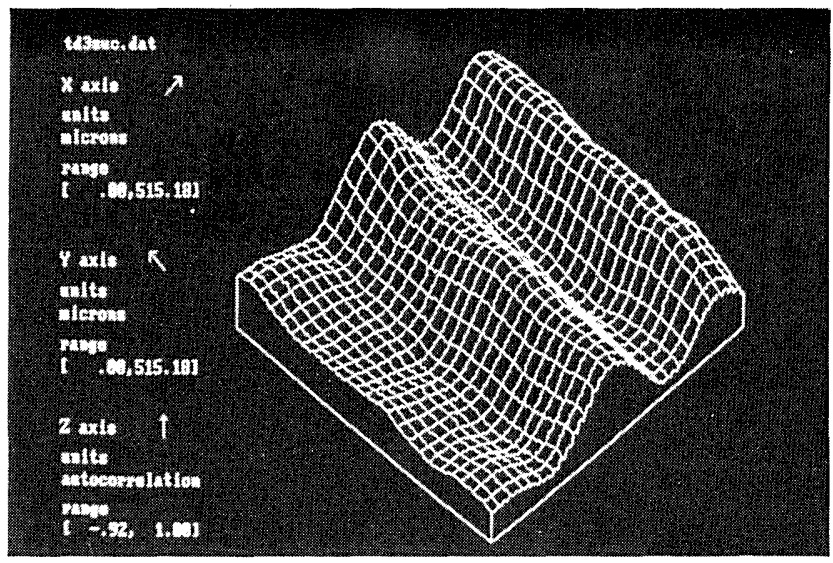

Fig. 8. Autocorrelation function of surface of Fig. 7.

intensity subtraction operations performed on the image of Fig. 3. Figure 6 shows the symbolic fringe extrema image derived from the processed interferogram. The surface calculated from Figs. 5 and 6 and its autocorrelation function are shown in Figs. 7 and 8. Numerical descriptors calculated for this surface are as follows: 
image size: $662 \mu \mathrm{m}$ square;

pixel size: $2.6 \mu \mathrm{m}$ square;

range in height $=448 \mu \mathrm{m}$;

rms roughness $=60 \mu \mathrm{m}$;

average roughness $=49 \mu \mathrm{m}$;

skew of the height distribution $=0.53$;

kurtosis of the height distribution $=2.9$;

autocorrelation (100 samples):

maximum $=1.0$ at $(0,0)$,

minimum $=-0.83$ at $(92,84) \mu \mathrm{m}$.

These results demonstrate the unique measurement capabilities afforded by projection interferometric techniques. Stylus profilometer measurements were attempted on this surface but were unsuccessful due to the steep slopes of the groove edges. Other optical surface roughness characterization techniques, such as measurement of total integrated scatter, or the angular distribution at scatter, would probably be unsuccessful on this surface due to its large average roughness.

\section{Conclusion}

The projection interferometer with digital analysis should prove to be a viable nondestructive method for contouring optically rough surfaces. Although the method is equally applicable to optical surfaces, the lack of dependence of the technique on surface reflectivity allows the application to materials previously unmeasurable by reflection interferometric methods. In addition, the high spatial bandwidth obtainable, limited by coherent speckle effects, allows the mapping of surface contours which cannot be measured by physical probes such as contact styli or capacitance probes. The technique is also applicable to surfaces with greater roughness than optical raster-scan methods, which are generally restricted to the measurement of roughnesses of the order of microns. Aside from the advantage of more general applicability, the greatest advantage which the projection interferometer has over other rough surface profilometers is the fact that it yields a 3-D output rather than a linear or point-by- point measurement. This facilitates measurement of the 2-D autocorrelation function of a surface, for example, which would be extremely difficult to duplicate with a scanning measurement system.

We are very grateful to Norman S. Eiss for providing stylus profilometer calibrations of our samples. In addition, S.P.A. and O.D.D.S. wish to thank the LusoAmerican Educational Commission for a grant supporting this collaborative research.

\section{References}

1. S. H. Rowe and W. T. Welford, "Surface Topography of Nonoptical Surfaces by Projected Interference Fringes," Nature London 216, 786 (1967)

2. W. T. Welford, "Some Applications of Projected Interference Fringes," Opt. Acta 16, 331 (1969).

3. S. H. Rowe, "Projected Interference Fringes in Holographic Interferometry," J. Opt. Soc. Am. 61, 1599 (1971).

4. A. J. MacGovern, "Projected Fringes and Holography," Appl. Opt. 11, 2972 (1972).

5. K. Leonhardt, "The Interference of Two Obliquely Intersecting Beams," Optik 41, 344 (1974).

6. O. D. D. Soares, "Non-optical Surface Topography by Projected Interference Fringes," Port. Phys. 13, 217 (1982).

7. O. D. D. Soares and S. P. Almeida, "Projection Interference Microscope," Proc. Soc. Photo-Opt. Instrum. Eng. 429, 32 (1983).

8. S. P. Almeida, R. W. Wygant, L. M. Bernardo, and O. D. D. Soares, "Analysis of Microscopic Surfaces by Projection Interference Fringes," J. Opt. Soc. Am. A 3(13), P42 (1986).

9. R. W. Wygant, S. P. Almeida, and O. D. D. Soares, "Surface Microtopography by Automated Processing of Projected Interference Fringes," Proc. Soc. Photo-Opt. Instrum. Eng. 42, 863 (1987).

10. R. W. Wygant, S. P. Almeida, and O. D. D. Soares, "Surface Micro-Metrology by Image Processing of Patterns from Collimated Interference Fringe Illumination," in OPTICSECOOSA, Birmingham, U.K., 22-25 Mar. 1988.

11. S. P. Almeida, R. W. Wygant, and O. D. D. Soares, "Automatic Surface Analysis with Fringe Projection," Proc. Soc. Photo-Opt. Instrum. Eng. 08, 952 (1988).

12. N. George, "About Speckle," Proc. Soc. Photo-Opt. Instrum. Eng. 5, 153 (1983).

\section{These times demand APPLIED OPTICS.}

Have you renewed your subscription? 